\title{
A Theory Relating Focal Epidemics to Crop-Weed Interactions
}

\author{
J. Frantzen and H. Müller-Schärer
}

Botanical Department, University of Fribourg, 3, rue Albert-Gockel, 1700 Fribourg, Switzerland. Accepted for publication 5 December 1997.

Phytopathology is concerned with the prevention of crop losses due to pathogens in agricultural plant populations and with plant population biology regarding the understanding of the causes of changes in numerical abundance and gene frequency in wild plant populations (1). In line with this separation of the two research fields, pathosystems were divided by Robinson (12) into artificial (crops) and natural (wild plants) pathosystems. Pathogens with weeds as hosts can neither be classified as artificial nor as natural and, therefore, a weed pathosystem may be distinguished and defined as any subsystem of an ecosystem that involves parasitism causing diseases of weeds (4). In general, characteristics of a weed pathosystem involve the occurrence of host and pathogen in a relatively uniform physical environment strongly influenced by humans and spatial variability due to both spacing and developmental stage of the host.

Knowledge of weed pathosystems is of interest for developing biological weed control using indigenous pathogens (7). Three approaches of biological control were distinguished by Charudattan (2): (i) the inoculative approach, (ii) the augmentative approach, and (iii) the inundative approach. The inoculative approach or classical approach is based on the innate ability of a pathogen to build up epidemics after introduction of a small amount of inoculum in an area where the target weed is present, but the pathogen was absent until the time of introduction. In contrast, the inundative or bioherbicide approach is based on the use of native pathogens that are applied onto the whole weed population. This knockdown approach requires relatively large amounts of infectious units of a pathogen. The augmentative approach may be viewed as the middle between the inoculative and inundative approach in terms of the amount of inoculum used and the management actions necessary to create epidemics.

The contrasts between the augmentative approach at one side and the inoculative and the inundative approach at the other side remained a bit vague. The augmentative approach was, therefore, redefined recently by Müller-Schärer and Frantzen (8) as the system management approach. The approach excludes the use of exotic organisms (classical approach) and the use of mass amounts of inoculum applied like a herbicide to the whole weed population (bioherbicide approach). The system management approach is intended not only for extensive agroecosystems, but also for intensive agroecosystems. The aim of the system management approach is less to eradicate a weed as to manage the weed pathosystem and so reducing competition exerted by the weed on a crop.

Stimulating epidemics and reducing competitiveness of the target weed are the key factors of the system management approach. In the following, we will present first a theory to dovetail these two key factors and, subsequently, the development of theory into

Corresponding author: J. Frantzen; E-mail address: jozef.frantzen@unifr.ch

Publication no. P-1997-1229-010

(C) 1998 The American Phytopathological Society practice will be illustrated by an epidemiological study directed to the model weed pathosystem Senecio vulgaris L.-Puccinia lagenophorae Cooke.

\section{THEORY}

An infected plant, or a few infected plants, may act as a primary focus inside a plant population from which a focal epidemic may start (17). Such a focal epidemic may proceed after some time with a constant, predictable speed (15). Employing the system management approach of biological weed control, the time required to build up an epidemic should fit in the time window for control of a weed. For example, vegetable crops may tolerate weeds at the early stages of growth, followed by a critical period in which weeds cause crop losses, and then, a final period in which weeds are again tolerated (11).

To dovetail the development of epidemics to crop losses caused by a weed population, we may consider the following equation (14)

$$
A(t)=\pi R^{2}(t)=\pi(\varepsilon+c t)^{2}
$$

in which $A$ is the area occupied by a focus at a certain time $(t), R$ is the radius of the focus, $t$ is the time a focus expands at a constant velocity $(c)$, and $\varepsilon$ is a constant correcting for an initial period of focus development. The assumption is that the focus is rotationally symmetric. Equation 1.1 may be rewritten as

$$
A(t)=\pi R^{2}(t)=\pi\left[f\left(t_{1}\right)+c\left(t-t_{1}\right)\right]^{2}
$$

in which $f$ is the function describing the development of the focus expansion during the period $t_{1}$ until focus expansion arrives at the constant velocity $(c)$, and $t-t_{1}$ is the period of focus expansion at constant velocity. For biological weed control we fill in $A(t)$ as the area of the weed to be controlled by a focus at the time a crop reaches the critical period. After setting this limit, the available time can be divided into the time required for focus development $\left(t_{1}\right)$ and the time for expansion of the focus at constant velocity $\left(t-t_{1}\right)$. The function $f\left(t_{1}\right)$ has not yet been described. A more formal description of this function is required.

The constant velocity $c$ can be determined from epidemics as van den Bosch et al. (14) did, but also can be calculated using an analytical model $(15,16)$. The calculation using an analytical model is based on the gross reproduction $\gamma \mathrm{S}_{0}$, a time kernel $i(t)$, and a contact distribution $D$. The gross reproduction is defined as the total number of daughter individuals (e.g., infected plants) produced by a mother individual. The time kernel represents the generation time and includes the infectious period and latent period. The contact distribution represents the spatial distribution of individuals and is determined by the dispersal gradient of dispersal units. The gross reproduction, the time kernel, and the contact distribution can be calculated from a field experiment in which only one generation of the pathogen is involved and, so, the velocity of focus expansion. In contrast, determining the velocity of focus expansion from epidemics may require more generations and, so, relatively large field experiments. 
We've assumed that an epidemic is focal and the focus expands after some time at a constant velocity. These assumptions can, and should be, checked in a field experiment as van den Bosch et al. (14) did. However, the function describing the spore-dispersal gradient also indicates whether a focus may develop symmetric and at a constant velocity (13). The spore dispersal gradient can be determined in a small-scale field experiment (described below).

\section{DATA}

Material and methods. The annual plant species S. vulgaris (groundsel) is considered as a weed in horticulture, vegetable crops, orchards, and vineyards (8). The rust fungus $P$. lagenophorae infects $S$. vulgaris plants by way of aecidiospores. Aecidia develop after infection and these produce aecidiospores, which infect again $S$. vulgaris plants.

Aecidial infection has a broad spectrum of impact on groundsel plants depending on biotic and abiotic conditions (10). Plants may be killed or, the other extreme, infection has no detectable impact on the host. In an experimental study, biomass losses of lettuce due to competition with $S$. vulgaris plants could be minimized by inoculation of the $S$. vulgaris plants with P. lagenophorae (9).

In the current study, three maternal half-sibling $S$. vulgaris lines were used and provided by G. S. Wyss (Swiss Federal Research Station, Wädenswil). The lines were named after their origin: (i) Unterehrendingen (Switzerland), (ii) Leiden (the Netherlands), and (iii) Lancaster (United Kingdom). Aecidiospores of one P. lagenophorae line were used. This line 'Unterehrendingen' was collected as an isolate from the same site as the plant line 'Unterehrendingen,' plants of the line 'Unterehrendingen' were inoculated with the isolate, and spores of one aecidium were used for subsequent multiplication of the rust on plants of the line 'Unterehrendingen' (G. S. Wyss, personal communication). Seeds and aecidiospores used in the experiments were collected from plants in a climate room with 16-h days (approximately $150 \mu \mathrm{mol} \mathrm{m} \mathrm{m}^{-2} \mathrm{~s}^{-1}$ at $23^{\circ} \mathrm{C}$ and $60 \%$ relative humidity) and 8 -h nights (at $15^{\circ} \mathrm{C}$ and $80 \%$ relative humidity). Seeds and spores from one generation were used in the experiments.

Twelve plots, each $2.7 \mathrm{~m}$ by $2.7 \mathrm{~m}$, were marked on a slightly inclined part of the university garden. Plots were separated by an approximately 20 -cm-wide row of plants of Lonicera pileata Oliv. to minimize dispersal of spores between plots. Plants of the perennial L. pileata were planted in March 1995. The height of the plants was approximately $20 \mathrm{~cm}$. If necessary, plots were weeded by hand and slugs were controlled chemically.

Wind speed and wind direction were measured using equipment of Étrélec S.A. (Renens, Switzerland) at about $1 \mathrm{~m}$ height at the center of the site, in 10-min intervals in 1995 and in 30-min intervals in 1996. Temperature and relative humidity were measured at 8-h intervals at about 10 and $40 \mathrm{~cm}$ above the soil at the center of the site using a Grant 1200 Squirrel (Grant Instruments, Cambridge, United Kingdom).

In 1995 , the central part of each plot $(1.10 \mathrm{~m}$ by $1.10 \mathrm{~m})$ was used and this part was divided into $11 \times 11$ cells. The size of a cell was $10 \mathrm{~cm}$ by $10 \mathrm{~cm}$. The central cell was kept free and one seed was placed in each of the other 120 cells by the end of April. Forty seeds of each of the three plant lines were randomly assigned to the 120 cells per plot. On 19 May (day 0), the following three treatments, each replicated in four plots, were applied randomly to the 12 plots: (i) whole plots were sprayed with an aecidiospore suspension of $P$. lagenophorae $(0.5 \mathrm{mg}$ of spores per $\mathrm{ml}$ of distilled water); (ii) a rust-infected, sporulating plant of the line 'Unterehrendingen' was placed in the central cell; and (iii) plots served as control, i.e., no inoculation. The plots of the treatments ii and iii were covered by plastic during the application of the spore suspension to the plots of treatment i. Spraying the aecidiospore suspension resulted in a density of about 900 aecidiospores per $\mathrm{cm}^{2}$ of soil surface. After spraying the spore suspen- sion, the plots were covered by plastic for one night to assure a high humidity. Subsequently, plants in all plots were checked daily on infection. Presence of one or more sori was used as criterion to declare a plant infected. The experiment was stopped when plants in the control plots became infected at a considerable level.

Infection progress curves were estimated using the log-logistic equation (5)

$$
y=\frac{c}{1+\left(\frac{t}{\tau}\right)^{-b}}
$$

in which $y$ is the fraction of plants infected, $c$ is the upper asymptote of $y, b$ is the shape parameter, $t$ is time in days, and $\tau$ is the mid-infection time at which one-half of the estimated maximum fraction of plants infected was infected. Curves were fitted to the data by nonlinear regression using SPSS (SPSS Inc., Chicago). The rate of infection $(d y / d t)$ at the mid-infection time $(\tau)$ was computed as

$$
v=(c \times b) /(4 \times \tau)
$$

Subsequently, the spatial pattern of infection incidence at the mid-infection time $(\tau)$ was studied using the Moran statistic for autocorrelation (6):

$$
I=\frac{\sum_{i j} w_{i j} z_{i} z_{j}}{W \sum_{i=1}^{n} z_{i}^{2}}
$$

and

$$
z_{i}=x_{i}-\bar{x} \text { and } z_{j}=x_{j}-\bar{x}
$$

in which $I$ is the Moran statistic with a value between -1 and $+1, n$ is the number of cells of a plot occupied by a plant, $w_{i j}$ a weight that defines two cells $i$ and $j$ as neighbors or not, $W$ is the sum of the weights, $x_{i}$ is the value of a variable in cell $i$, and $x_{j}$ is the value of a variable in cell $j$. In the current study, the variable was a plant either infected (value $=1$ ) or not (value $=0$ ). Cells were defined as neighbors by distance, i.e., the four cells adjacent to the sides of a cell (Rook's move) and the four cells adjacent to the corners of a cell (Bishop's move). Whether the $I$ value differed significantly from zero (a spatially random distribution) was tested by a randomization test.

The spatial pattern of infection incidence within a plot at the mid-infection time was graphically expressed as infection probabilities by means of kriging using a Geostatistics software package (Gamma Design Software, Plainwell, MI).

In 1996, a plot at the center of the site was selected to determine spore dispersal. The spore traps used are constructed to determine sedimentation of spores and have been described previously (6). The diameter of a spore trap is $6 \mathrm{~cm}$. Spore traps were placed at $15,30,45,60$, and $75 \mathrm{~cm}$ from the center of the plot. The center of the spore trap was used as point of reference. Spore traps were placed in the wind directions 0 (north), 45, 90, 135, 180, 225, 270, and 315 degrees. A sporulating plant with a foliage diameter of about $10 \mathrm{~cm}$ was placed at the center of the plot on 17 July (day 0) at 8.00 a.m. Spores of $P$. lagenophorae were trapped during three consecutive $24-\mathrm{h}$ periods. The number of spores was determined by counting the number of spores trapped on an area of $0.32 \mathrm{~cm}^{2}$ at the center of a spore trap. Two models were fitted to the data (3)

$$
\begin{gathered}
y=a \cdot x^{-b} \\
y=c \cdot \exp (-d \cdot x)
\end{gathered}
$$

in which $a$ and $c$ are constants related to the source strength, and $b$ and $d$ are parameters indicating the steepness of the spore-dispersal gradient. Equation 4.1 is the power law model and equation 4.2 is the exponential model. Models were fitted to the data by nonlinear regression using SPSS (SPSS Inc.). 
Results. Not all seeds emerged, and so, cells remained empty, resulting in gaps in the experimental $S$. vulgaris populations. Depending on the plot, a fraction (0.7 to 0.85$)$ of the seeds emerged. The mean of the fraction of seeds emerged per plot was similar for the three plant lines. At day 0 , the date of spraying plots with a spore suspension and transfer of an inoculum plant to the center of plots, the number of true leaves present differed among plants and was in the range of 0 to 4 . The height of the plants was in the range of 5 to $15 \mathrm{~cm}$.

Control plots remained relatively free of infection. The mean of the fraction of plants infected per plot was 0.003 at day 14 and 0.028 at day 17 , at which day the experiment was stopped. In the plots treated with an aecidiospore suspension, infection was observed on some plants at day 10. The mean of the fraction of plants infected per plot increased to 0.93 at day 14 .

The infection progress in each of the plots with an inoculum plant was described well by the log-logistic equation (Fig. 1). The $R^{2}$ was 0.99 or higher for the curves fitted. Estimates of parameter $b$ were less accurate than those of the parameters $c$ and $\tau$ (Table 1). The parameter $c$ of the curve of plot 10 had to be constrained to 1 . The epidemic in this plot was delayed compared with the other plots, and this delay was expressed in a higher value of $\tau$.

The spatial distribution of infected plants at the mid-infection time $\tau$ was nonrandom in the four plots with an inoculum plant, as indicated by the Moran statistic. Depending on the plot, the values were 0.15 to 0.35 and all four values differed significantly $(P<$ $0.001)$ from zero. The probability of infection at the mid-infection time decreased with distance from the center of a plot (Fig. 2A, B, $\mathrm{C}$, and D). Figure 2 is a smoothed presentation of the field situations in which cells were present without plants and plants were infected (1) or not (0). However, Figure 2 shows quite well the places where the highest fractions of plants infected were observed at the mid-infection time and the places where the lowest fractions of plants infected were observed, i.e., a high probability versus a low probability of infection. The slightly higher abundance of infection in the southern parts of the plots was in agreement with the prevailing wind from the north on days 0 to 4 . Un-

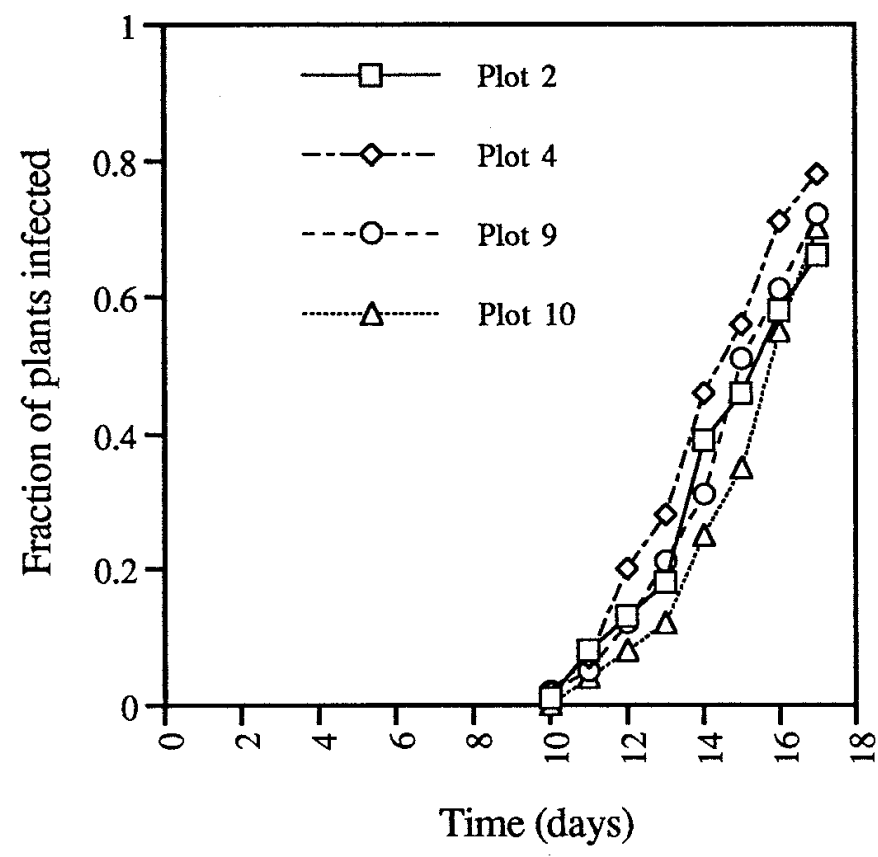

Fig. 1. Infection progress curves observed in four plots in 1995. Day 0 is the day of transfer of an inoculum plant to the center of each plot. Fraction of plants infected is expressed as the number of diseased plants present at a certain day divided by the total number of plants present in a plot by the end of the experiment (day 17). fortunately, cables of the data-logger were damaged at this time, hindering wind measurement on the subsequent days.

In the plot used to determine spore dispersal, deposition of spores decreased with distance from the center of the plot (Fig. 3). The number of spores trapped during the first $24 \mathrm{~h}$ (days 0 to 1 ) was higher than during the following two 24-h periods. Withering of the inoculum plant was observed and sporulation stopped. A slight west orientation was observed in spore trapping that did not correspond to the prevailing wind direction measured at $1 \mathrm{~m}$ height (described above). Fitting the power law model to the data of the first 24-h trapping period resulted in estimates of $a=159,120$ (standard error of 11,507) and $b=2.34$ (standard error of 0.03 ), and the $R^{2}$ was 0.999 . Fitting the exponential model to the data of the first 24-h trapping period resulted in estimates of $c=1282$ (standard error of 149) and $d=0.102$ (standard error of 0.007 ), and the $R^{2}$ was 0.997 . The curve based on the exponential model approaches the $\mathrm{x}$ axis more at the distances indicated, i.e., the curve is exponentially bound, than the curve based on the power law model, i.e., the curve is not exponentially bound.

\section{DISCUSSION AND CONCLUSIONS}

The experiment in 1995 was set up to follow the spread of the rust from one inoculum plant, i.e., one generation. Plots with an inoculum plant were used to follow this spread. The control plots were used to monitor inoculum coming from outside the field or inocuum passing between the plots. The data on infection incidence in the control plots suggested that the data obtained from the plots with an inoculum plant were not seriously confounded by inoculum from outside the plot until day 17, the day the experiment was finished. Results of the spatial analysis agree with a spread of the rust from the inoculum plant at the center of a plot. The data of infection incidence in the plots sprayed with a spore suspension indicated a latent period of at least 10 days, and so, we are sure that epidemic spread within one generation was followed in the plots with an inoculum plant.

Calculation of the constant velocity of focus expansion requires the gross reproduction $\gamma \mathrm{S}_{0}$, the time kernel $i(t)$, and the contact distribution $D$ as written in the Theory section. The set up of the 1995 and 1996 experiment should enable this calculation, but some problems were encountered.

The disease progress curves of the plots with an inoculum plant suggested that spread of disease was not yet finished at the time the experiment was finished. So, it was not possible to determine the maximum number of plants that could be infected by aecidiospores from the inoculum plant, i.e., the gross reproduction. An experiment with a better protection of plots from inoculum from outside is required to determine the gross reproduction.

TABLE 1. Estimates and standard error (in parentheses) of parameters of a $\log$-logistic model ${ }^{\mathrm{w}}$ fitted to data of infection of Senecio vulgaris plants by Puccinia lagenophorae in four experimental plots ${ }^{\mathrm{x}}$

\begin{tabular}{lcrrc}
\hline & \multicolumn{4}{c}{ Parameter } \\
\cline { 2 - 5 } Plot & $c$ & \multicolumn{4}{c}{$\tau(d)^{\mathrm{y}}$} & \multicolumn{1}{c}{$b$} & $v\left(d^{-1}\right)^{\mathrm{y}, \mathrm{z}}$ \\
\hline 2 & $0.79(0.09)$ & $14.3(0.5)$ & $9.5(1.6)$ & 0.13 \\
4 & $0.93(0.07)$ & $14.1(0.3)$ & $9.1(1.0)$ & 0.15 \\
9 & $0.92(0.08)$ & $14.8(0.3)$ & $9.5(0.9)$ & 0.15 \\
10 & $1.00(0.15)$ & $15.7(0.5)$ & $10.2(1.3)$ & 0.16 \\
\hline $\mathrm{w}$ & $y=\frac{c}{1+\left(\frac{t}{\tau}\right)^{-b}}$
\end{tabular}

in which $y=$ the fraction of plants infected, $c=$ the upper asymptote of $y, b=$ the shape parameter, $t=$ time in days, and $\tau=$ the mid-infection time at which one-half of the estimated maximum fraction of plants infected was infected.

${ }^{x}$ An inoculum plant was placed at the center of each plot. Data is from 1995. ${ }^{y} d=$ number of days.

$\mathrm{z}$ The rate of infection $(d y / d t)$ at the mid-infection time $(\tau)$ was computed as $v=$ $c \times b /(4 \times \tau)$. 
The latent period could be determined approximately from the plots sprayed with a spore suspension and was in the range of 10 to 14 days. The rust-infected plant used to determine the sporedispersal gradient in 1996 wilted after 3 days, suggesting a rather short infectious period. We assume wilting resulted from the transfer of the infected plant from the climate room to the field, and the infectious period estimated may not be representative for natural rust infection. More information about the infectious period is required to determine the time kernel $i(t)$.

The spore-dispersal gradient was determined, and both the power law and exponential model fitted well to the data of spore dispersal. The power law is not exponentially bound, and Shaw (13) demon-
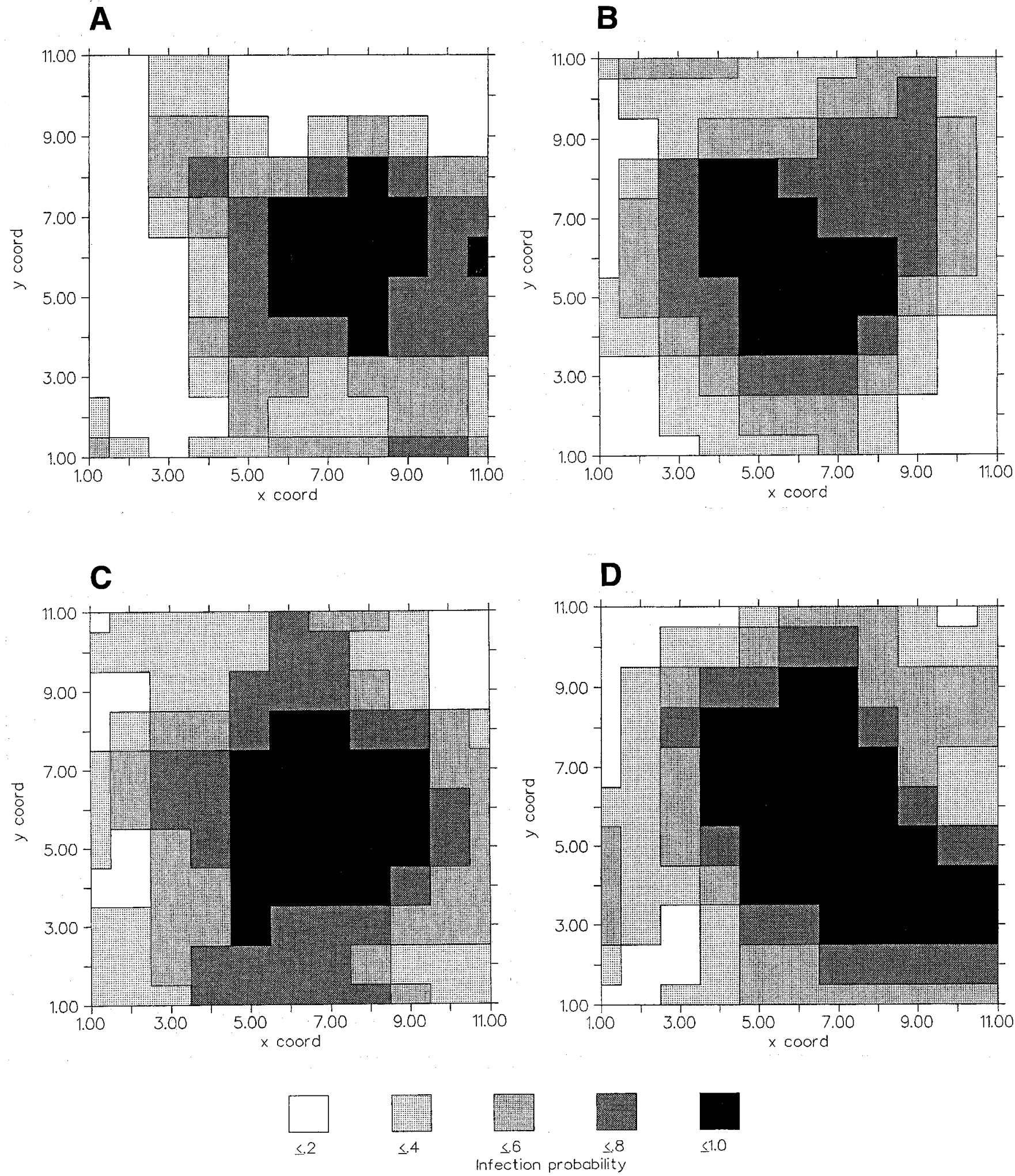

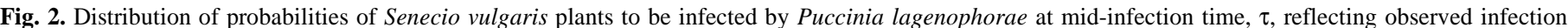

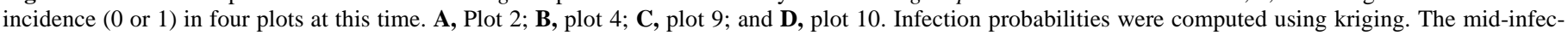

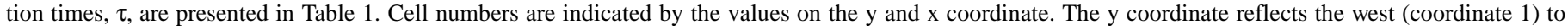
east (coordinate 11) direction, and the x coordinate the north (coordinate 1) to south (coordinate 11) direction. Data is from 1995. 


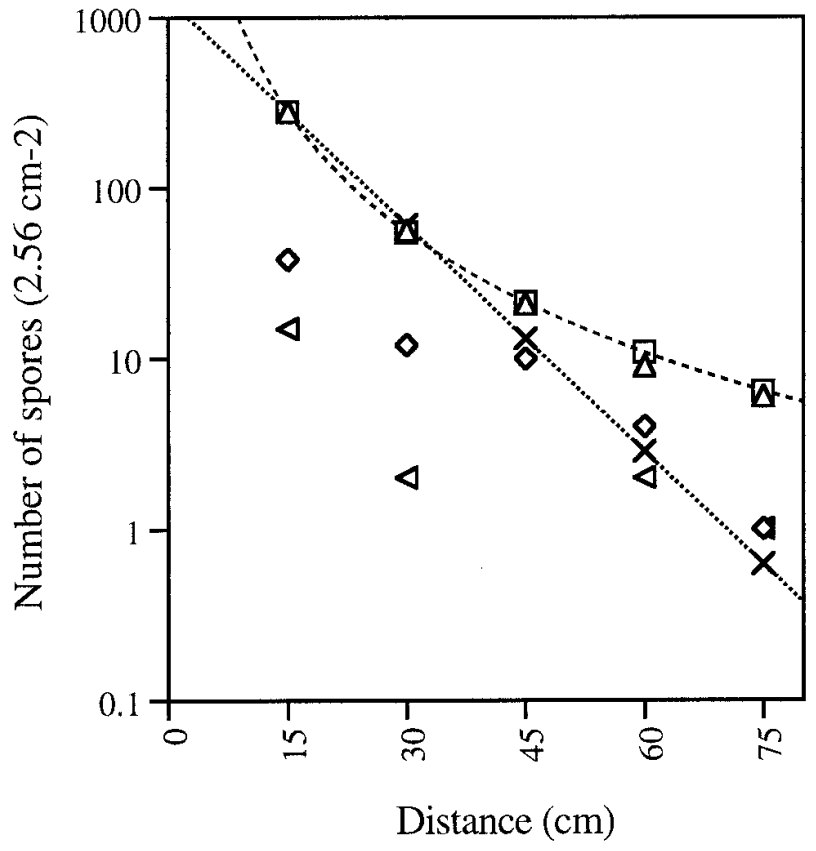

$\Delta \quad$ Observed day $0-1$

Observed day $1-2$

$\varangle \quad$ Observed day $2-3$

- - Power law fitted to day 0-1

$\times \quad$..... Exponential fitted to day 0-1

Fig. 3. Deposition of Puccinia lagenophorae aecidiospores related to distance from the stem of a sporulating plant. Spores were trapped during three subsequent periods of $24 \mathrm{~h}$. Entries are sums of spores counted in eight directions, i.e., eight spore traps. A power law and exponential model was fitted to the data of the first period. Days refer to the days in the experiment. Data is from 1996.

strated that functions that are not exponentially bound do not result in a constant wave of focus expansion. In contrast, dispersal gradients described by exponentially bound functions do result in a constant wave of focus expansion. So, the distinction between the power law model and the exponential model is relevant to predicting epidemics and employment of the system management approach of biological weed control. A spore-dispersal gradient described by the exponential model fits in the theory developed here, whereas one described by the power law model does not. The data presented of the weed pathosystem $S$. vulgaris- $P$. lagenophorae did not allow a clear-cut choice between the two models. A more precise estimation of the tail of the spore-dispersal gradient is required.
Equation 1.2 is a framework for the system management approach of biological weed control. Experiments on a rather large scale are required to determine whether or not focus expansion arrives at a constant velocity and, if so, how much time is required to arrive at the constant velocity. Experiments at a rather small scale can already provide much information, but problems may be encountered as shown by the example of the S. vulgaris-P. lagenophorae pathosystem. The function $f\left(t_{1}\right)$ needs to be explored to arrive at a formal description of this function.

\section{ACKNOWLEDGMENTS}

This study was part of the European COST-project 816. We thank the Swiss Ministry of Education and Science for their financial support. We thank M. van Ooijen, J. Wallinga, and two anonymous referees for their comments on a previous version of the manuscript and S. Bollinger of the Botanical Garden for her support.

\section{LITERATURE CITED}

1. Antonovics, J., and Alexander, H. M. 1989. The concept of fitness in plant-fungal pathogen systems. Pages 185-214 in: Plant Disease Epidemiology. 2. Genetics, Resistance, and Management. K. J. Leonard and W. J. Fry, eds. McGraw-Hill, New York.

2. Charudattan, R. 1988. Inundative control of weeds with indigenous fungal pathogens. Pages 86-110 in: Fungi in Biological Control Systems. Manchester University Press, Manchester, England.

3. Fitt, B. D. L., and McCartney, H. A. 1986. Spore dispersal in relation to epidemic models. Pages 311-345 in: Plant Disease Epidemiology. 1. Population Dynamics and Management. K. J. Leonard and W. J. Fry, eds. Macmillan Publishing Company, New York.

4. Frantzen, J. 1994. Studies on the weed pathosystem Cirsium arvensePuccinia punctiformis. Ph.D. thesis. University of Wageningen, Wageningen, the Netherlands. ISBN 90-5485-211-9.

5. Frantzen, J. 1994. The effect of temperature on the germination of teliospores of Puccinia punctiformis. Phytopathology 84:1043-1046.

6. Frantzen, J. 1994. An epidemiological study of Puccinia punctiformis (Str.) Röhl as a stepping-stone to the biological control of Cirsium arvense (L.) Scop. New Phytol. 127:147-154.

7. Hasan, S., and Ayres, P. G. 1990. The control of weeds through fungi: Principles and prospects. New Phytol. 115:201-222.

8. Müller-Schärer, H., and Frantzen, J. 1996. An emerging system management approach for biological weed control in crops: Senecio vulgaris as a research model. Weed Res. 36:483-491.

9. Paul, N. D., and Ayres, P. G. 1987. Effects of rust infection of Senecio vulgaris on competition with lettuce. Weed Res. 27:431-441.

10. Paul, N. D., Ayres, P. G., and Hallett, S. G. 1993. Mycoherbicides and other biocontrol agents for Senecio spp. Pestic. Sci. 37:323-329.

11. Roberts, H. A. 1976. Weed competition in vegetable crops. Ann. Appl. Biol. 83:321-347.

12. Robinson, R. A. 1976. Plant Pathosystems. Springer Verlag, Berlin.

13. Shaw, M. W. 1995. Simulation of population expansion and spatial pattern when individual dispersal distributions do not decline exponentially with distance. Proc. R. Soc. Lond. B 259:243-248.

14. van den Bosch, F., Frinking, H. D., Metz, J. A. J., and Zadoks, J. C. 1988. Focus expansion in plant disease. III: Two experimental examples. Phytopathology 78:919-925.

15. van den Bosch, F., Zadoks, J. C., and Metz, J. A. J. 1988. Focus expansion in plant disease. I: The constant rate of focus expansion. Phytopathology 78:54-58.

16. van den Bosch, F., Zadoks, J. C., and Metz, J. A. J. 1988. Focus expansion in plant disease. II: Realistic parameter-sparse models. Phytopathology 78:59-64.

17. Zadoks, J. C., and Schein, R. D. 1979. Epidemiology and Plant Disease Management. Oxford University Press, New York. 